\title{
Management of toxicities associated with targeted therapies for HR-positive metastatic breast cancer: a multidisciplinary approach is the key to success
}

\author{
Marina Elena Cazzaniga ${ }^{1}$ (D) Romano Danesi ${ }^{2} \cdot$ Corrado Girmenia $^{3} \cdot$ Pietro Invernizzi $^{4} \cdot$ Alessandra Elvevi $^{4}$. \\ Massimo Uguccioni ${ }^{5}$ on behalf of NetworkER+
}

Received: 11 February 2019 / Accepted: 26 April 2019 / Published online: 7 May 2019

(c) The Author(s) 2019

\begin{abstract}
Purpose Agents targeting HR-positive, HER2-negative locally advanced or metastatic breast cancer have improved patient outcomes compared with conventional single-agent endocrine therapy. Currently, approved targeted agents include everolimus and three CDK4/6 inhibitors, palbociclib, ribociclib, and abemaciclib. Unlike the well-characterized and easily manageable safety profile of endocrine therapies, adverse events associated with targeted therapies are complex and potentially severe. Their prompt recognition and treatment, crucial for prolonged endocrine sensitivity and survival, may be challenging and requires a multidisciplinary effort and a good knowledge of drug interactions.

Methods We reviewed the current evidence on the drug safety of targeted agents for metastatic breast cancer currently used in clinical practice in Italy, supported by the clinical experience of Italian oncologists with expertise in the field.

Results All oncologists had used CDK4/6 inhibitors in clinical practice and/or within a clinical trial. The clinical management of toxicities, including dose adjustments, treatment interruptions, and concerns regarding special populations is discussed, and the management of relevant adverse events, related to individual agents and class-specific, toxicities is reviewed. Hematologic toxicities have the greatest impact on clinical management of the disease and on patients. Although toxicities associated with the new treatments result in more visits to the physician and more time and attention with patients, they are manageable, with no need for the oncologist to consult with specialist physicians.

Conclusions Based on the available evidence and current guidelines, we propose a series of practical recommendations for multidisciplinary clinical management of the various toxicities associated with the addition of targeted agents to endocrine therapy.
\end{abstract}

Keywords Abemaciclib $\cdot$ Advanced breast cancer $\cdot$ Everolimus $\cdot$ Neutropenia $\cdot$ Palbociclib $\cdot$ Ribociclib

\section{Introduction}

The members of the NetworkER+ Study Group are listed in Acknowledgement section.

Marina Elena Cazzaniga

marina.cazzaniga@asst-monza.it

1 Department of Medical Oncology \& Phase 1 Research Centre ASST-Monza, Via Pergolesi 33, 20900 Monza, Italy

2 Unit of Clinical Pharmacology and Pharmacogenetics, Department of Clinical and Experimental Medicine, University of Pisa, Pisa, Italy
The development of targeted agents for the treatment of hormone receptor (HR)-positive, HER2-negative locally advanced or metastatic breast cancer has significantly improved progression-free survival (PFS) and rates of objective response

3 Department of Hematology, Oncology and Dermatology, Azienda Policlinico Umberto I, Sapienza University, Rome, Italy

4 Division of Gastroenterology and Center for Autoimmune Liver Diseases, Department of Medicine and Surgery, University of Milan Bicocca, Monza, Italy

5 Cardiology 1/CCU, San Camillo Hospital, Rome, Italy 
and clinical benefit compared with conventional single-agent endocrine therapy [1-8]. Currently, approved targeted agents include everolimus, an oral inhibitor of the mammalian target of rapamycin (mTOR) [9] and three oral selective inhibitors of cyclin-dependent kinases (CDK) 4 and 6, palbociclib, ribociclib, and abemaciclib [10-12].

Compared with the well-characterized and easily manageable safety profile of endocrine therapies, usually related to symptoms typically associated with estrogen deprivation, including arthralgia, hot flashes, and fatigue, adverse events associated with targeted therapies are distinct and more complex. Results from the registration trials have shown that combining targeted agents with endocrine therapies substantially increases the incidence of grade 3-4 adverse events compared with conventional single-agent endocrine therapy $[1,2,4,5$, 7, 8]. Everolimus has been associated with stomatitis and noninfectious pneumonitis, while neutropenia is the most common toxicity related to CDK4/6 inhibitors [13-18]. Prompt recognition and management of these toxicities is vital for treatment persistence and, therefore, for maximizing survival. However, this may pose a challenge to many oncologists, as experience in clinical practice with novel treatment strategies for metastatic breast cancer is still limited. In addition, for fear of adverse events, many oncologists may not prescribe targeted therapies to elderly or frail patients, precluding them from effective treatment options $[14,19]$. Another issue of importance for optimal use of strategies combining endocrine therapy and targeted agents is knowledge of drug interactions related to everolimus and CDK4/6 inhibitors.

In the present paper, we review the relevant evidence concerning drug interactions and safety profiles of everolimus and the CDK4/6 inhibitors in use in clinical practice in Italy for the treatment of HR-positive metastatic breast cancer and discuss the toxicities associated with this novel approach to the treatment of breast cancer. Based on the available evidence and current guidelines, we propose a series of practical recommendations for clinical management of the various toxicities associated with the addition of targeted agents to endocrine therapy.

\section{Methods}

A multidisciplinary panel of five Italian clinicians, including an oncologist, a pharmacologist, a hematologist, a gastroenterologist, and a cardiologist, convened at a meeting organized within the NetworkER+ project (held in Rome, Italy in January 2018). A group of 20 oncologists from the HERMIONE network also attended the meeting. HERMIONE is a network platform that was launched in February 2017 to promote communication and collaboration among oncology centers and oncologists involved in the treatment of metastatic HR-positive breast cancer in Italy.
The main objectives of the NetworkER+ meeting were to: review current evidence concerning the drug interactions and safety profile of targeted agents used in metastatic breast cancer; produce recommendations for the optimal management of drug interactions and toxicities related to targeted agents; focus on frail patients with metastatic breast cancer, namely the elderly and those with organ dysfunction.

\section{Clinical experience}

The 20 oncologists at the meeting were from Oncology Units treating $\geq 150$ breast cancer cases per year, across the entire national territory. All had used CDK4/6 inhibitors in their clinical practice; $60 \%$ within a clinical trial. In their experience, hematologic toxicities have the greatest impact on both clinical management of the disease and on patients. The toxicities associated with the new treatments result in more visits to the physician and in more time and attention devoted to patients. However, they are considered to be manageable, with no need for the oncologist to consult with specialist physicians.

\section{Drug interactions}

Drug interactions are an important cause of morbidity. The most relevant pharmacokinetic interactions involve drugs that can inhibit or induce enzymes in the hepatic cytochrome P450 system [16]. Members of the CYP3A family of P450 enzymes are probably the most important of all drug-metabolizing enzymes because of their abundance and ability to process a large number of chemically unrelated drugs from almost every drug class [16]. Strong CYP3A4 enzyme inhibitors in clinical use include the antibiotics clarithromycin, telithromycin, and erythromycin, and the antifungal agents ketoconazole and voriconazole; grapefruit juice is also a strong CYP3A4 inhibitor. Strong CYP3A4 inducers include the antibiotic rifampicin and antiepileptic agents such as barbiturates, phenytoin, and carbamazepine; herbal preparations (St. John's wort) can also induce CYP3A4. Predicting drug interactions is not easy. A factor that further complicates the understanding of drug interactions is the finding that inhibitors/inducers and substrates of CYP3A4 overlap with those of the drug-transporter protein P-glycoprotein (P-gp) [16]. P-gp acts as an efflux pump-exporting drugs, for example, into the intestinal lumen.

Everolimus and the CDK4/6 inhibitors palbociclib, ribociclib, and abemaciclib are administered orally and have different half-lives, undergo predominantly hepatic metabolism, and are substrates of CYP3A4; everolimus is also a substrate of P-gp (Table 1) [20-23]. The concomitant use of everolimus or a CDK4/6 inhibitor with inhibitors of CYP3A4 (or P-gp for everolimus) may, therefore, result in 
an increased plasma concentrations and pharmacological effect of the targeted agent, while their concomitant use with an inducer of CYP3A4 (or P-gp for everolimus) may lead to lower plasma concentrations and decreased pharmacological effect. According to the labels of the four targeted agents, the concomitant use of strong CYP3A4 inhibitors or inducers is not recommended (everolimus) or should be avoided (CDK4/6 inhibitors). If unavoidable, the dose of everolimus or CDK4/6 inhibitors should be adjusted as recommended (Table 2). Liver function tests should be performed before initiating treatment with abemaciclib and ribociclib and repeated every 2 weeks for the first two cycles, monthly for the next two cycles, then as clinically indicated $[10,12]$. An additional relevant interaction exists for ribociclib with anti-arrhythmic drugs and other medications that prolong the QT interval on the electrocardiogram (ECG). Such medications should be avoided in patients receiving ribociclib. Ribociclib is also a strong to moderate inhibitor of CYP3A4 and should be used with caution with CYP3A4 substrates that have a narrow therapeutic index. With the exception of palbociclib, the targeted agents can be taken regardless of food intake. Palbociclib should be taken with food, as its absorption and drug exposure were shown to be low in the fasted state [11, 24].

Overall, to minimize the risk of harm due to drug interactions, it is important to be aware of possible interactions. It is imperative to ask patients about the use of other medications, including herbal products. At the same time, patients should be informed about drug interactions and instructed to not take any additional medication (including over-thecounter and herbal products) during treatment with targeted therapies without first consulting with the oncologist. Recommended measures to reduce the risk of adverse effects include using an alternative treatment, adjustment of the dose of targeted agent, and close patient monitoring.

\section{Safety data from clinical trials}

Class-effect toxicities associated with the use of mTOR inhibitors include stomatitis, which encompasses inflammation and ulceration of the oral mucosal lining, non-infectious pneumonitis (a non-malignant inflammatory infiltration of the lung), infections, and metabolic adverse events (hyperglycemia and hyperlipidemia) [13].

According to a recent meta-analysis of six randomized controlled trials (RCTs) investigating CDK4/6 inhibitors in combination with an aromatase inhibitor or fulvestrant in women with advanced breast cancer, the most frequent adverse event (all grades) in the group treated with the combination of CDK4/6 inhibitor plus endocrine therapy was neutropenia $(65 \%)$ followed by diarrhea $(49 \%)$, infections (44\%), nausea (40\%), fatigue (39\%), and leukopenia (35\%) [25]. Other safety issues reported in clinical trials include hepatobiliary toxicity (ribociclib, abemaciclib), prolongation of the QT interval on ECG (ribociclib), and venous thromboembolism (abemaciclib) [10, 12].

\section{Stomatitis and pneumonitis}

In the BOLERO-2 trial, everolimus-related toxicities included stomatitis, pneumonitis, and hyperglycemia [26]. These toxicities were generally of mild or moderate severity and, with the exception of pneumonitis, occurred early after everolimus initiation (within 8 weeks). Adverse events were generally manageable with dose reduction and interruption for a median duration of 7 days. Of note, appropriate dose reductions for toxicity did not have a negative impact on efficacy [27]. In a meta-analysis of five randomized, doubleblind phase three clinical trials of everolimus in patients with solid tumors, including breast cancer, renal cell carcinoma, carcinoid tumors, and pancreatic neuroendocrine tumors, the rate of stomatitis was $67 \%$ [16]. Stomatitis was mostly grade $1-2$, with grade $3-4$ reported in $<10 \%$ of patients. Stomatitis did not adversely affect PFS. Of note, prophylactic use of a steroid mouthwash has been shown to substantially reduce the incidence and severity of stomatitis in patients undergoing combination therapy with everolimus and an aromatase inhibitor [28].

\section{Gastrointestinal toxicity}

Cases of severe gastrointestinal (GI) bleeding during targeted therapy with everolimus have been reported [29-31]. In two of these reports, gastric antral vascular ectasia (GAVE) was identified as the likely cause of bleeding [29, 30]; GI bleeding was successfully treated with endoscopic hemostasis using argon plasma coagulation after treatment discontinuation. In another case, GI bleeding was associated with treatment initiation and resolved following discontinuation [31]. Endoscopy revealed that the bleeding was secondary to erosive gastritis, and several endoscopic interventions were needed to achieve hemostasis.

The rapidly proliferating GI epithelium is one of the most vulnerable tissues to the effects of antiproliferative drugs. Indeed, GI events including nausea, vomiting, and diarrhea are shared by most anticancer drugs. A meta-analysis of four studies with CDK4/6 inhibitors (palbociclib, ribociclib) was performed to assess the risk of GI toxicities associated with CDK4/6 inhibitors [32]. Adding CDK4/6 inhibitors to endocrine therapy was found to marginally increase the incidence of any-grade decreased appetite, nausea, vomiting, and diarrhea with no significant increase in the risk of high-grade GI toxicities compared with control. In the MONARCH 2 study, which demonstrated that abemaciclib significantly extends PFS when added to fulvestrant in women with advanced breast cancer whose disease had progressed while 


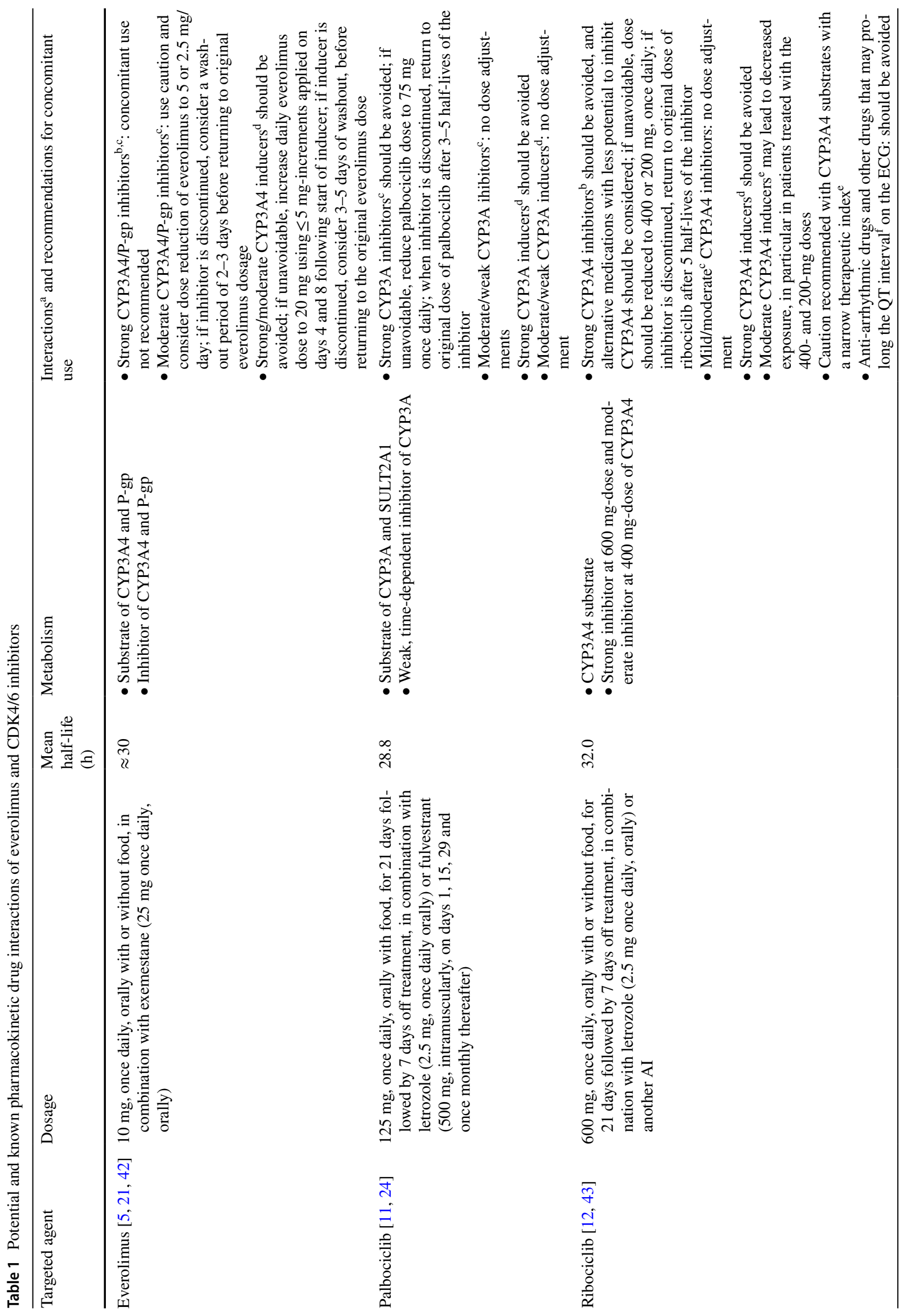


on endocrine therapy, the most common adverse events in the abemaciclib-fulvestrant versus fulvestrant alone arms were diarrhea $(86.4 \%$ vs. $24.7 \%)$, neutropenia $(46.0 \%$ vs. $4.0 \%)$, nausea $(45.1 \%$ vs. $22.9 \%)$ and fatigue $(39.9 \%$ vs. $26.9 \%$ ) [6]. In most cases, diarrhea was effectively managed using supportive treatment, such as antidiarrheal medications and/or dose adjustments. Diarrhea was also the most frequent adverse event in the MONARCH-3 trial investigating abemaciclib plus a nonsteroidal aromatase inhibitor (NSAI) as initial therapy in postmenopausal women with advanced breast cancer [3]. Diarrhea was reported by $81.3 \%$ of patients, but was mostly of grade 1 (44.6\%). The most frequent grade 3 or 4 event was neutropenia ( $21.1 \%$ vs. $1.2 \%$ in the group with a NSAI alone), diarrhea (9.5\% vs. $1.2 \%)$, and leukopenia (7.6\% vs. $0.6 \%$ ). Low-grade diarrhea was managed in most cases with conventional antidiarrheal medications and dose adjustment.

\section{Neutropenia}

The frequencies of CDK4/6 inhibitor-related grade 3-4 neutropenia reported in RCTs were highest with palbociclib (54-66\%, PALOMA-2 and 3 trials) and ribociclib (27-59\%, MONALEESA-2 trial) and lowest with abemaciclib (21-27\%, MONARCH-2 and 3) [1-3, 6, 18, 33-35]. According to a detailed safety analysis of the PALOMA-3 trial comparing fulvestrant plus palbociclib versus fulvestrant alone (median follow-up, 8.9 months), neutropenia was the most common grade $3(55 \%)$ and $4(10 \%)$ adverse event in patients receiving combination therapy [18]. However, febrile neutropenia was reported only in $0.9 \%$ and $0.6 \%$ of patients treated with fulvestrant-palbociclib and fulvestrant alone, respectively. Dose modifications for grade 3-4 neutropenia did not compromise PFS. The consequences of myelosuppression experienced during palbociclib treatment are different from those associated with chemotherapy-induced myeloablation [18]. The neutropenia associated with CDK4/6 inhibitors is effectively and rapidly managed by dose delay, interruption, or reduction and without the use of granulocyte colony-stimulating factors (G-CSF). This suggests that mature white blood cells are present in the bone marrow and can rapidly enter the blood circulation when drug levels decrease [18]. In contrast, chemotherapy results in the destruction of progenitor cells leading to more persistent and severe neutropenia.

\section{Elevation of liver enzymes}

The CDK4/6 inhibitors, ribociclib and abemaciclib, have been associated with hepatobiliary toxicity [3, 4, 34, 35]. In the MONALEESA-2 study, grade 3 or 4 elevations in alanine and aspartate aminotransferase levels were reported 
Table 2 Dose modifications recommended for the management of adverse reactions associated with targeted therapies

\begin{tabular}{lllll}
\hline Agent & Starting dose & First reduction & Second reduction & Third reduction \\
\hline Everolimus & $10 \mathrm{mg}$ once daily & $5 \mathrm{mg}$ once daily & Not applicable & Not applicable \\
Palbociclib & $125 \mathrm{mg}$ once daily & $100 \mathrm{mg}$ once daily & $75 \mathrm{mg}$ once daily & Not applicable \\
Ribociclib & $600 \mathrm{mg}$ once daily & $400 \mathrm{mg}$ once daily & $200 \mathrm{mg}$ once daily & Not applicable \\
Abemaciclib & & & \\
$\begin{array}{l}\text { Combination therapy } \\
\text { Monotherapy }\end{array}$ & $150 \mathrm{mg}$ twice daily & $100 \mathrm{mg}$ twice daily & $50 \mathrm{mg}$ twice daily & Not applicable \\
& $200 \mathrm{mg}$ twice daily & $150 \mathrm{mg}$ twice daily & $100 \mathrm{mg}$ twice daily & $50 \mathrm{mg}$ twice daily
\end{tabular}

in $9.3 \%$ and $5.7 \%$, respectively, of patients receiving ribociclib plus letrozole versus letrozole alone and have also been observed with other CDK4/6 inhibitors in combination with aromatase inhibitors. Most cases of liver-enzyme elevation were asymptomatic and reversed by dose adjustment. A detailed analysis of the safety and health-related quality of life from the MONALEESA-2 trial confirmed that hepatobiliary toxicity was more common in patients treated with ribociclib plus letrozole than in those treated with letrozole alone, with the onset of such events more common during the first 12 months of treatment $(21.3 \%$ vs. $9.5 \%$ for ribociclib plus letrozole vs. letrozole alone) [34].

Abemaciclib was also associated with a higher rate of hepatic transaminase elevations in the abemaciclib arm of the MONARCH-3 trial [3]. These were generally manageable with dose reduction or dose omission and were resolved with drug discontinuation.

\section{QTc prolongation}

Some drugs can cause a delay in cardiac repolarization, measured as prolongation of the QT interval on ECG. Delay in cardiac repolarization can increase the risk of cardiac arrhythmias, most notably torsades de pointes [3]. In the MONALEESA-2 trial comparing ribociclib combined with letrozole versus letrozole alone for first-line treatment of postmenopausal women with advanced breast cancer, the most frequent adverse event in the ribociclib arm was neutropenia (74.3\% vs. $5.2 \%$ in the letrozole alone arm), followed by nausea (51.5\% vs. $28.5 \%)$, infections ( $50.3 \%$ vs. $42.4 \%)$, fatigue $(36.5 \%$ vs. $30.0 \%)$, and diarrhea (35.0\% vs. $22.1 \%)$ [33]. These events were mostly grade 1 or 2 . An increase of more than $60 \mathrm{~ms}$ from baseline in the QTcF (QT corrected by Fridericia) interval occurred in nine patients $(2.7 \%)$ in the ribociclib group and in no patients in the placebo group. In the second interim analysis of the MONALEESA-2 trial (at a median follow-up of 26.4 months), safety results were comparable with those reported in the first analysis [4]. A > $60 \mathrm{~ms}$ prolongation from baseline in the QT interval occurred in ten patients $(3.0 \%)$ in the ribociclib plus letrozole group and one patient $(0.3 \%)$ in the letrozole group. A total of $12(3.6 \%)$ patients receiving ribociclib plus letrozole had at least one QT of $>480 \mathrm{~ms}$ versus two patients $(0.6 \%)$ in the control arm; in the ribociclib plus letrozole group, 11 of these patients had been reported at the time of the first analysis. Two of the 12 patients in the ribociclib plus letrozole group had received a concomitant prohibited medication with a known risk to prolong QT; three of the 12 patients had dose interruption, but this was due to QT prolongation only in 1 patient. No arrhythmia (torsades de pointes) was reported in the ribociclib group. Together these findings confirm that ECG changes observed with targeted therapies are mostly asymptomatic and that QT prolongations are managed effectively by adjusting the ribociclib dose.

\section{Clinical management of toxicities}

Dose adjustments and/or temporary interruption due to adverse reactions are recommended for all targeted agents based on individual safety and tolerability [9-12]. Table 2 shows the dose modifications recommended in the labels of everolimus, palbociclib, ribociclib, and abemaciclib. Of note, the labels state that if further dose reduction is required below the lowest dose indicated, treatment should be permanently discontinued. Overall, dose adjustment is usually not required for adverse reactions of grade 1, while treatment should be discontinued when the adverse reactions are grade 4. For grade 2 and 3 adverse reactions, temporary treatment interruption should be considered until symptoms improve to grade $\leq 1$. When this improvement is achieved, treatment should be resumed at the next lower dose. For example, in patients developing grade 2 everolimus-related non-infectious pneumonitis, everolimus should be interrupted until symptoms improve to grade $\leq 1$; treatment should be reinitiated at $5 \mathrm{mg}$ daily. If symptoms fail to improve within 4 weeks, treatment should be permanently discontinued. The same is recommended for grade 3 non-infectious pneumonitis; in this case, if toxicity recurs at grade 3 after resuming treatment at $5 \mathrm{mg}$ daily, everolimus should be permanently discontinued. If patients develop grade 2 stomatitis, everolimus should be interrupted until recovery to grade $\leq 1$ and reinitiated at the same dose; if stomatitis recurs at grade 2 , treatment should be interrupted until recovery to grade $\leq 1$ and reinitiated at $5 \mathrm{mg}$ daily. In the case of grade 3 stomatitis, everolimus should be interrupted until recovery to grade $\leq 1$ and resumed at $5 \mathrm{mg}$ daily. The approach to 
management of other relevant safety issues related to targeted therapies is discussed in detail below.

\section{Management of neutropenia and prevention of infections}

Neutropenia induced by CDK4/6 inhibitors is reversible and can be readily managed by dose interruption or modification without compromising treatment efficacy. The propensity to develop higher-grade neutropenia during treatment with CD4/6 inhibitors can generally be recognized within the first months of treatment [18]. Appropriately tailored dose adjustments should be promptly implemented to reduce the risk of recurrent episodes of severe neutropenia and/or febrile neutropenia [18]. It is, therefore, important to monitor absolute neutrophil counts early during treatment so that timely dose adjustments can be implemented in patients experiencing grade 3-4 neutropenia.

Figure 1 shows the recommended algorithm for the management of neutropenia associated with palbociclib, ribociclib, and abemaciclib [10-12]. Options for management of neutropenia and infections include dose reduction or drug interruption. Patients who develop grade 2 neutropenia during treatment have an increased risk of developing highergrade neutropenia and febrile neutropenia. They should, therefore, be closely monitored (complete blood count) throughout treatment with CDK4/6 inhibitors. As for infections secondary to neutropenia, antibacterial prophylaxis should be administered only if grade 4 neutropenia persists for $>7$ days and particularly in the presence of mucositis. Vaccination against Streptococcus pneumoniae before initiating therapy and annual influenza vaccination is recommended for all patients. The American Society of Clinical Oncology (ASCO) guidelines recommend hepatitis B virus (HBV) screening in all cancer patients treated with conventional chemotherapy or targeted therapies [36]. Based on the serological profile of HBV infection, a different therapeutic strategy will be needed (i.e., treatment or prevention of viral reactivation) [37].

\section{Management of diarrhea, nausea, and vomiting}

In the absence of signs of infection, diarrhea should generally be managed using non-pharmacologic interventions, including hydration, appropriate diet, and avoidance of
Fig. 1 Management of CDK4/6-related neutropenia as recommended in the labels of palbociclib, ribociclib, and abemaciclib. For the recommended dose adjustments, please refer to Table 2. ANC absolute neutrophil count, $C B C$ complete blood count. *The label of abemaciclib does not differentiate between grade 3 neutropenia with or without fever $>38.5{ }^{\circ} \mathrm{C}$ and/or infection. Reproduced with permission from Spring et al. [17]

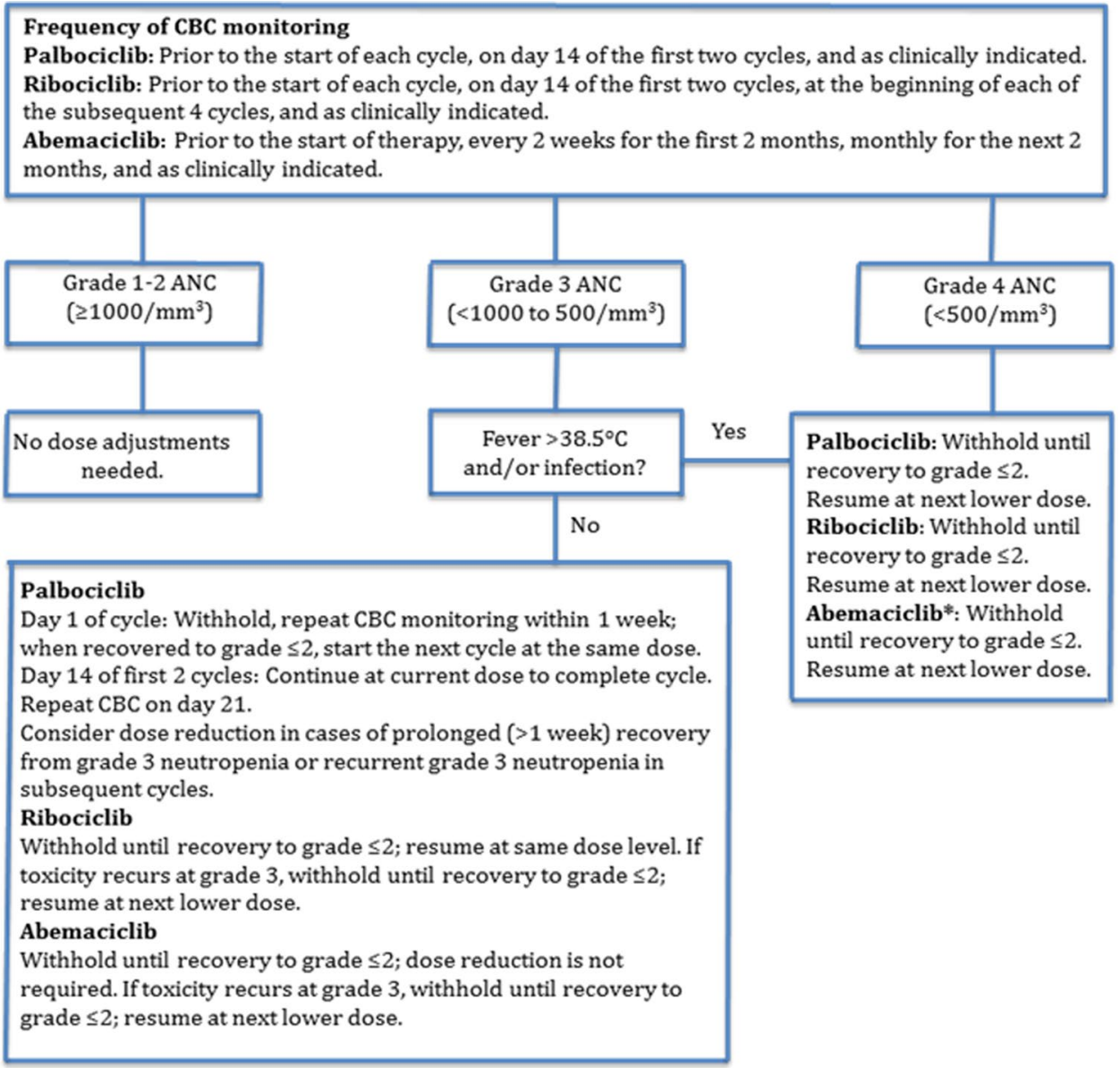

Palbociclib: Prior to the start of each cycle, on day 14 of the first two cycles, and as clinically indicated. Ribociclib: Prior to the start of each cycle, on day 14 of the first two cycles, at the beginning of each of the subsequent 4 cycles, and as clinically indicated.

Abemaciclib: Prior to the start of therapy, every 2 weeks for the first 2 months, monthly for the next 2 months, and as clinically indicated.

Grade 1-2 ANC

Fever $>38.5^{\circ} \mathrm{C}$

and/or infection?

No

Palbociclib

Day 1 of cycle: Withhold

Day 14 of first 2 cycles: Continue at current dose to complete cycle.

Repeat $C B C$ on day 21.

entropent grade 3 neutropenia in

Ribociclib

Withhold until recovery to grade $\leq 2$; resume at same dose level. If toxicity recurs at grade 3 , withhold until recovery to grade $\leq 2$;

required. If toxicity recurs at grade 3 , withhold until recovery to

grade $\leq 2$; resume at next lower dose. 
diarrhea-inducing agents. However, in the case of abemaciclib, the Summary of Product Characteristics directs that treatment with antidiarrheal agents, such as loperamide, should be started at the first sign of loose stools [10]. Recurrent or high-grade diarrhea requires dose reduction. Antidiarrheal medication (loperamide) can also be used. Nausea and vomiting should be treated with antiemetics, including metoclopramide, prochlorperazine, haloperidol, or serotonin-receptor antagonists as needed. Caution should be taken when prescribing symptomatic therapies because of potential drug interactions (Table 1). Particular attention is needed with the concomitant administration of ribociclib with antiemetics (e.g., intravenous ondansetron, dolasetron, metoclopramide, diphenhydramine, haloperidol) because of the risk of QT interval prolongation [38, 39]. With regards to palbociclib, rabeprazole (a proton pump inhibitor) decreases its serum concentration and $\mathrm{H} 2$-receptor antagonists or locally acting antacids should be used for the management of nausea. Dexamethasone and aprepitant may, respectively, decrease or increase serum levels of palbociclib; possible alternatives are metoclopramide and domperidone [32].

\section{QT interval prolongation}

Prolongation of the QT interval has been frequently associated with cancer therapies, with or without targeted agents [40]. Substantial prolongation of QT (>500 $\mathrm{ms})$ is more frequent with targeted therapies [40]. Of note, the incidence of major arrhythmias and myocardial infarction caused by therapy-related QT prolongation is very low [40]. Patients at risk of QT prolongation or with QT prolongation before or during cancer therapy should be assessed as outlined in Fig. 2 [40]. In cancer patients, altered electrolyte levels (hypokalemia, hypocalcemia, and hypomagnesemia) are often due to reduced electrolyte intake, diarrhea, vomiting, fever with sweating, use of laxatives, and therapy with steroids. Furthermore, the use of drugs with potentially synergic effects on QT prolongation (Table 1) and structural cardiomyopathy can also contribute to QT prolongation. A cardiologist should be consulted in the following cases: QT prolongation $>500 \mathrm{~ms}$; prolonged QT during treatment and presence of symptoms of heart disease; history of arrhythmias; history of presyncope or syncope with a likely cardiac origin; prolonged QT and bradycardia $<60 \mathrm{bpm}$ (these patients should undergo ECG monitoring in a cardiology unit).

When initiating treatment with ribociclib, the following points should be kept in mind: (1) QT $>500 \mathrm{~ms}$, severe arrhythmias, or sudden cardiac death related to targeted therapies are very rare; (2) At the first manifestation of QT prolongation, a manual measurement should be performed; (3) If prolonged QT is confirmed, reversible electrolyte alterations should be excluded; (4) Treatment with ribociclib can be initiated in patients with QT < $450 \mathrm{~ms}$; (5) Avoid concomitant administration of drugs that can prolong the QT; (6) ECG should be measured at baseline, 14 days from the beginning of treatment, and at the beginning of the second cycle of treatment; (7) Electrolyte levels should be measured before initiating treatment and if there is an indication for additional monitoring.

\section{Treatment of elderly and frail patients}

A significant proportion of patients with breast cancer are aged $\geq 65$ years. Management of toxicities related to targeted therapies may be particularly challenging in this age group because of the presence of comorbidities and frailty. Due to the frequent use of polytherapy by older patients, the management of drug interactions may be particularly complex. Older patients are generally underrepresented in oncology clinical trials, including those that have investigated targeted therapies for HR-positive, HER2-negative metastatic breast cancer [41]. Subgroup analyses and a recent systematic review have shown that adding everolimus or a CDK4/6 inhibitor to endocrine therapy is, however, a feasible strategy in patients aged $\geq 65$ years, resulting in improved survival, response rates, and clinical benefit [19, 27, 41]. In these analyses, the safety profile of everolimus and CDK4/6 inhibitors was consistent with that observed for the overall study population [41]. As a consequence, according to the prescribing information, there is no need for dose adjustments of everolimus and CDK4/6 inhibitors in patients aged $\geq 65$ years, based exclusively on age [9-12]. Subgroup analyses have also shown that the toxicities induced by targeted therapies in older patients were effectively managed by dose interruptions or reductions, as in the overall population [19].

Based on published evidence, current guidelines, and personal experience, we strongly recommend initiating targeted therapy with CDK4/6 inhibitors in elderly patients at the full licensed dose. Close monitoring to ensure the early detection of adverse events is highly recommended, as toxicities may rapidly worsen in older individuals. Currently, no data are available regarding the use of CDK 4/6 inhibitors in patients with organ dysfunction; these patients should be managed as recommended in the labels of the various targeted agents.

\section{Conclusions}

The addition of targeted agents, everolimus, or CDK4/6 inhibitors to endocrine therapy has considerably improved the outcomes of patients with metastatic HR-positive, HER2-negative breast cancer. This strategy is, however, associated with an increased risk of class-specific toxicities that are potentially serious. Most adverse events associated with targeted therapies have an early onset and 


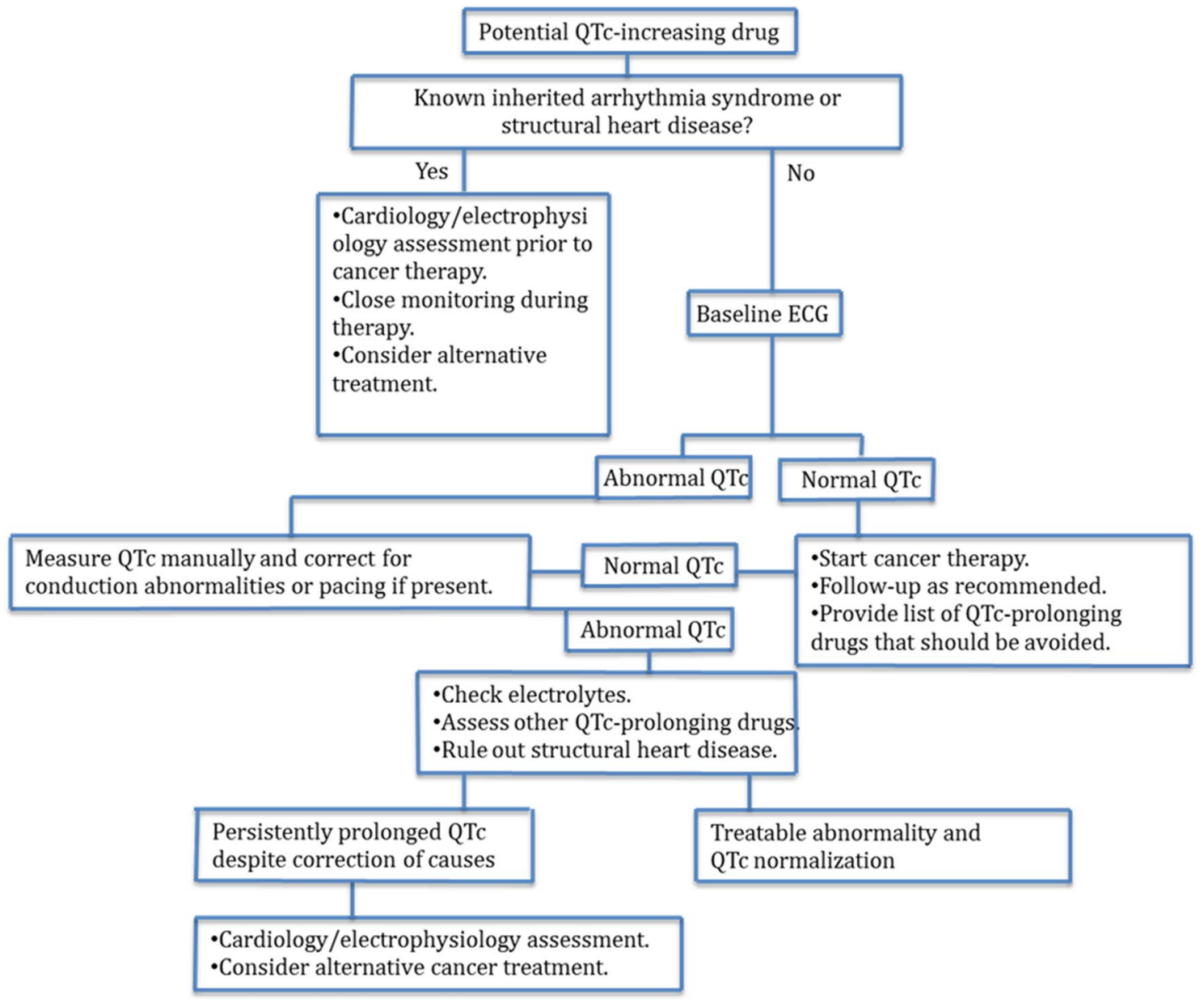

Fig. 2 Assessment of patients at risk of QTc prolongation or with QTc prolongation before or during cancer treatment. Reproduced with permission from Porta-Sanchez et al. [40]

can generally be readily managed by dose adjustments and temporary interruptions (Box 1). Early treatment of adverse events is crucial. In clinical practice, the optimal implementation of targeted therapies will require a multidisciplinary effort, the ability to stratify patients according to the risk of adverse events, treatment individualization, and close patient monitoring. Efforts aimed at increasing patient awareness of treatment-related toxicities will also be required.

\section{Box 1 Summary of main recommendations for management of toxicities associated with targeted therapies}

- Where possible, strategies to prevent expected adverse events should be considered (e.g., prophylactic use of steroid mouthwashes to prevent stomatitis; vaccinations to prevent common infections).

- Early management of toxicities is very important, and thus the prompt recognition of signs and symptoms is crucial. Patients should be informed about treatmentrelated toxicities.

- Dose reductions for grade 2-3 adverse events are feasible in many instances, with no detrimental effect on efficacy. Grade 1 toxicities usually do not require dose modifications, while grade 4 toxicities should prompt permanent treatment discontinuation. 
- Careful consideration of drug interactions (CYP3A4 inhibitors/inducers, as well as QT-prolonging drugs) is required when prescribing medications for the treatment of adverse events.

- The co-administration of strong inhibitors or inducers of CYP3A4 with everolimus and CDK4/6 inhibitors should be avoided. Alternative medications should be given (e.g., metoclopramide and domperidone as antiemetics; loperamide as antidiarrheal agent).

- Neutropenia induced by CDK4/6 inhibitors is reversible and can be readily managed by dose interruption or modification without compromising treatment efficacy, as described in the drug labels.

- Antidiarrheal agents, such as loperamide, should be started at the first sign of loose stools with abemaciclib; otherwise, diarrhea induced by CDK4/6 inhibitors should initially be treated with non-pharmacologic interventions; antiemetics can be used for nausea and vomiting, after evaluation of possible drug interactions.

- Liver function tests should be performed before initiating treatment with abemaciclib and ribociclib and liver function monitored throughout treatment.

- QT interval prolongation has been associated with targeted therapies; however, major arrhythmias and myocardial infarction have been rarely reported.

- Patients should be seen by a cardiologist when: QT prolongation is $>500 \mathrm{~ms}$; prolonged QT during treatment and presence of symptoms of heart disease; history of arrhythmias; history of presyncope or syncope with a likely cardiac origin; prolonged QT and bradycardia $<60$ bpm.

- Older patients ( $\geq 65$ years) can be treated with targeted therapies; the profile of adverse events is similar to that of younger patients. No dose adjustments are required based solely on age.

- Drug interactions need particular consideration in older patients on polytherapy.

Acknowledgements We wish to thank Dr. Monica Perez Gila (Clinical Research Coordinator-Oncologia Medica A.S.S.T. Monza-San Gerardo) for her assistance to the HERMIONE network. We also thank Health Publishing \& Services who provided editorial assistance prior to submission.

List of the 20 oncologists from the HERMIONE network who contributed their clinical experience: Laura Amaducci-Faenza; Francesco Atzori-Cagliari; Livio Blasi-Palermo; Chiara Butti-Gallarate (MI); Elena Collovà-Legnano (MI); Enrico De Conciliis-Asti; Alessandra Fabi-Roma; Antonio Febbraro-Benevento; Ornella GarroneCuneo; Lorenzo Gianni-Rimini; Francesco Giotta-Bari; Nicla La Verde-Milano; Andrea Michelotti-Pisa; Raffaella Palumbo-Pavia; Ida Paris-Roma; Mirco Pistelli-Ancona; Laura Pizzuti- Roma; Daniela Rubino-Bologna; Maria Rosaria Valerio-Palermo; Fable Zustovich-Belluno.
Author contributions MEC Conception/design. RD, CG, PI, AE, MU, MEC Provision of study material or patients. RD, CG, PI, AE, MU, MEC Collection and/or assembly of data. MEC, RD, MU Data analysis and interpretation. MEC Manuscript writing. RD, CG, PI, AE, MU Final approval of manuscript

Funding Novartis Farma Italy provided financial support for editorial assistance. No Grant number applicable.

\section{Compliance with ethical standards}

Ethical approval This article does not contain any studies with human participants or animals performed by any of the authors.

Conflict of interest The authors declare that they have no conflict of interest.

Open Access This article is distributed under the terms of the Creative Commons Attribution 4.0 International License (http://creativeco mmons.org/licenses/by/4.0/), which permits unrestricted use, distribution, and reproduction in any medium, provided you give appropriate credit to the original author(s) and the source, provide a link to the Creative Commons license, and indicate if changes were made.

\section{References}

1. Cristofanilli M, Turner NC, Bondarenko I, Ro J, Im SA, Masuda N, Colleoni M, DeMichele A, Loi S, Verma S, Iwata H, Harbeck N, Zhang K, Theall KP, Jiang Y, Bartlett CH, Koehler M, Slamon D (2016) Fulvestrant plus palbociclib versus fulvestrant plus placebo for treatment of hormone-receptor-positive, HER2negative metastatic breast cancer that progressed on previous endocrine therapy (PALOMA-3): final analysis of the multicentre, double-blind, phase 3 randomised controlled trial. Lancet Oncol 17(4):425-439. https://doi.org/10.1016/S1470-2045(15)00613-0

2. Finn RS, Martin M, Rugo HS, Jones S, Im SA, Gelmon K, Harbeck N, Lipatov ON, Walshe JM, Moulder S, Gauthier E, Lu DR, Randolph S, Dieras V, Slamon DJ (2016) Palbociclib and letrozole in advanced breast cancer. N Engl J Med 375(20):1925-1936. https://doi.org/10.1056/NEJMoa1607303

3. Goetz MP, Toi M, Campone M, Sohn J, Paluch-Shimon S, Huober J, Park IH, Tredan O, Chen SC, Manso L, Freedman OC, Garnica Jaliffe G, Forrester T, Frenzel M, Barriga S, Smith IC, Bourayou N, Di Leo A (2017) MONARCH 3: abemaciclib as initial therapy for advanced breast cancer. J Clin Oncol 35(32):3638-3646. https ://doi.org/10.1200/JCO.2017.75.6155

4. Hortobagyi GN, Stemmer SM, Burris HA, Yap YS, Sonke GS, Paluch-Shimon S, Campone M, Petrakova K, Blackwell KL, Winer EP, Janni W, Verma S, Conte P, Arteaga CL, Cameron DA, Mondal S, Su F, Miller M, Elmeliegy M, Germa C, O'Shaughnessy J (2018) Updated results from MONALEESA-2, a phase III trial of first-line ribociclib plus letrozole versus placebo plus letrozole in hormone receptor-positive, HER2-negative advanced breast cancer. Ann Oncol 29(7):1541-1547. https://doi. org/10.1093/annonc/mdy155

5. Royce M, Bachelot T, Villanueva C, Ozguroglu M, Azevedo SJ, Cruz FM, Debled M, Hegg R, Toyama T, Falkson C, Jeong J, Srimuninnimit V, Gradishar WJ, Arce C, Ridolfi A, Lin C, Cardoso F (2018) Everolimus plus endocrine therapy for postmenopausal women with estrogen receptor-positive, human epidermal growth factor receptor 2-negative advanced breast cancer: a clinical 
trial. JAMA Oncol 4(7):977-984. https://doi.org/10.1001/jamao ncol.2018.0060

6. Sledge GW Jr, Toi M, Neven P, Sohn J, Inoue K, Pivot X, Burdaeva O, Okera M, Masuda N, Kaufman PA, Koh H, Grischke EM, Frenzel M, Lin Y, Barriga S, Smith IC, Bourayou N, Llombart-Cussac A (2017) MONARCH 2: abemaciclib in combination with fulvestrant in women with HR +/HER2- advanced breast cancer who had progressed while receiving endocrine therapy. J Clin Oncol 35(25):2875-2884. https://doi.org/10.1200/ JCO.2017.73.7585

7. Tripathy D, Im SA, Colleoni M, Franke F, Bardia A, Harbeck N, Hurvitz SA, Chow L, Sohn J, Lee KS, Campos-Gomez S, Villanueva Vazquez R, Jung KH, Babu KG, Wheatley-Price P, De Laurentiis M, Im YH, Kuemmel S, El-Saghir N, Liu MC, Carlson G, Hughes G, Diaz-Padilla I, Germa C, Hirawat S, Lu YS (2018) Ribociclib plus endocrine therapy for premenopausal women with hormone-receptor-positive, advanced breast cancer (MONALEESA-7): a randomised phase 3 trial. Lancet Oncol 19(7):904-915. https://doi.org/10.1016/S1470-2045(18)30292-4

8. Yardley DA, Noguchi S, Pritchard KI, Burris HA 3rd, Baselga J, Gnant M, Hortobagyi GN, Campone M, Pistilli B, Piccart M, Melichar B, Petrakova K, Arena FP, Erdkamp F, Harb WA, Feng W, Cahana A, Taran T, Lebwohl D, Rugo HS (2013) Everolimus plus exemestane in postmenopausal patients with $\mathrm{HR}(+)$ breast cancer: BOLERO-2 final progression-free survival analysis. Adv Ther 30(10):870-884. https://doi.org/10.1007/s12325-013-0060-1

9. Everolimus summary of product characteristics. http://www.ema. europa.eu/docs/en_GB/document_library/EPAR_-_Product_Infor mation/human/001038/WC500022814.pdf. Accessed 31 Aug 2018

10. Abemaciclib summary of product characteristics. https://www. accessdata.fda.gov/drugsatfda_docs/label/2018/208855s0001bl. pdf. Accessed 31 Aug 2018

11. Palbociclib summary of product characteristics. http://www.ema. europa.eu/docs/en_GB/document_library/EPAR_-_Product_Infor mation/human/003853/WC500217196.pdf. Accessed 31 Aug 2018

12. Ribociclib summary of product characteristics. http://www.ema. europa.eu/docs/en_GB/document_library/EPAR_-_Product_Infor mation/human/004213/WC500233997.pdf. Accessed 31 Aug 2018

13. Aapro M, Andre F, Blackwell K, Calvo E, Jahanzeb M, Papazisis K, Porta C, Pritchard K, Ravaud A (2014) Adverse event management in patients with advanced cancer receiving oral everolimus: focus on breast cancer. Ann Oncol 25(4):763-773. https://doi. org/10.1093/annonc/mdu021

14. Cazzaniga ME, Airoldi M, Arcangeli V, Artale S, Atzori F, Ballerio A, Bianchi GV, Blasi L, Campidoglio S, Ciccarese M, Cursano MC, Piezzo M, Fabi A, Ferrari L, Ferzi A, Ficorella C, Frassoldati A, Fumagalli A, Garrone O, Gebbia V, Generali D, La Verde N, Maur M, Michelotti A, Moretti G, Musolino A, Palumbo R, Pistelli M, Porpiglia M, Sartori D, Scavelli C, Schirone A, Turletti A, Valerio MR, Vici P, Zambelli A, Clivio L, Torri V, E. V. A. Study Group (2017) Efficacy and safety of everolimus and exemestane in hormone-receptor positive $(\mathrm{HR}+)$ human-epidermal-growthfactor negative (HER2-) advanced breast cancer patients: new insights beyond clinical trials. The EVA study. Breast 35:115-121

15. Generali D, Montemurro F, Bordonaro R, Mafodda A, Romito S, Michelotti A, Piovano P, Ionta MT, Bighin C, Sartori D, Frassoldati A, Cazzaniga ME, Riccardi F, Testore F, Vici P, Barone CA, Schirone A, Piacentini F, Nole F, Molino A, Latini L, Simoncini EL, Roila F, Cognetti F, Nuzzo F, Foglietta J, Minisini AM, Goffredo F, Portera G, Ascione G, Mariani G (2017) Everolimus plus exemestane in advanced breast cancer: safety results of the BALLET study on patients previously treated without and with chemotherapy in the metastatic setting. Oncologist 22(6):648654. https://doi.org/10.1634/theoncologist.2016-0461

16. Rugo HS, Hortobagyi GN, Yao J, Pavel M, Ravaud A, Franz D, Ringeisen F, Gallo J, Rouyrre N, Anak O, Motzer R (2016) Metaanalysis of stomatitis in clinical studies of everolimus: incidence and relationship with efficacy. Ann Oncol 27(3):519-525. https ://doi.org/10.1093/annonc/mdv595

17. Spring LM, Zangardi ML, Moy B, Bardia A (2017) Clinical management of potential toxicities and drug interactions related to cyclin-dependent kinase 4/6 inhibitors in breast cancer: practical considerations and recommendations. Oncologist 22(9):10391048. https://doi.org/10.1634/theoncologist.2017-0142

18. Verma S, Bartlett CH, Schnell P, DeMichele AM, Loi S, Ro J, Colleoni M, Iwata H, Harbeck N, Cristofanilli M, Zhang K, Thiele A, Turner NC, Rugo HS (2016) Palbociclib in combination with fulvestrant in women with hormone receptor-positive/HER2-negative advanced metastatic breast cancer: detailed safety analysis from a multicenter, randomized, placebo-controlled, phase III Study (PALOMA-3). Oncologist 21(10):1165-1175. https://doi. org/10.1634/theoncologist.2016-0097

19. Sonke GS, Hart LL, Campone M, Erdkamp F, Janni W, Verma S, Villanueva C, Jakobsen E, Alba E, Wist E, Favret AM, Bachelot T, Hegg R, Wheatley-Price P, Souami F, Sutradhar S, Miller M, Germa C, Burris HA (2018) Ribociclib with letrozole vs letrozole alone in elderly patients with hormone receptor-positive, HER2negative breast cancer in the randomized MONALEESA-2 trial. Breast Cancer Res Treat 167(3):659-669. https://doi.org/10.1007/ s10549-017-4523-y

20. Flaherty KT, Lorusso PM, Demichele A, Abramson VG, Courtney R, Randolph SS, Shaik MN, Wilner KD, O’Dwyer PJ, Schwartz GK (2012) Phase I, dose-escalation trial of the oral cyclin-dependent kinase 4/6 inhibitor PD 0332991, administered using a 21-day schedule in patients with advanced cancer. Clin Cancer Res 18(2):568-576. https://doi.org/10.1158/1078-0432.CCR-11-0509

21. Grabowsky JA (2013) Drug interactions and the pharmacist: focus on everolimus. Ann Pharmacother 47(7-8):1055-1063. https:// doi.org/10.1345/aph.1R769

22. Infante JR, Cassier PA, Gerecitano JF, Witteveen PO, Chugh R, Ribrag V, Chakraborty A, Matano A, Dobson JR, Crystal AS, Parasuraman S, Shapiro GI (2016) A phase I study of the cyclin-dependent kinase 4/6 inhibitor ribociclib (LEE011) in patients with advanced solid tumors and lymphomas. Clin Cancer Res 22(23):5696-5705. https://doi.org/10.1158/1078-0432. CCR-16-1248

23. Tate SC, Sykes AK, Kulanthaivel P, Chan EM, Turner PK, Cronier DM (2018) A population pharmacokinetic and pharmacodynamic analysis of abemaciclib in a phase I clinical trial in cancer patients. Clin Pharmacokinet 57(3):335-344. https://doi.org/10.1007/s4026 2-017-0559-8

24. Ruiz-Garcia A, Plotka A, O'Gorman M, Wang DD (2017) Effect of food on the bioavailability of palbociclib. Cancer Chemother Pharmacol 79(3):527-533. https://doi.org/10.1007/s0028 0-017-3246-4

25. Ding W, Li Z, Wang C, Ruan G, Chen L, Tu C (2018) The CDK4/6 inhibitor in HR-positive advanced breast cancer: a systematic review and meta-analysis. Medicine (Baltimore) 97(20):e10746. https://doi.org/10.1097/MD.0000000000010746

26. Rugo HS, Pritchard KI, Gnant M, Noguchi S, Piccart M, Hortobagyi G, Baselga J, Perez A, Geberth M, Csoszi T, Chouinard E, Srimuninnimit V, Puttawibul P, Eakle J, Feng W, Bauly H, ElHashimy M, Taran T, Burris HA 3rd (2014) Incidence and time course of everolimus-related adverse events in postmenopausal women with hormone receptor-positive advanced breast cancer: insights from BOLERO-2. Ann Oncol 25(4):808-815. https://doi. org/10.1093/annonc/mdu009 
27. Pritchard KI, Burris HA III, Ito Y, Rugo HS, Dakhil S, Hortobagyi GN, Campone M, Csoszi T, Baselga J, Puttawibul P, Piccart M, Heng D, Noguchi S, Srimuninnimit V, Bourgeois H, Gonzalez MA, Osborne K, Panneerselvam A, Taran T, Sahmoud T, Gnant M (2013) Safety and efficacy of everolimus with exemestane vs. exemestane alone in elderly patients with HER2-negative, hormone receptor-positive breast cancer in BOLERO-2. Clin Breast Cancer 13(6):421-432. https://doi.org/10.1016/j.clbc.2013.08.011

28. Rugo HS, Seneviratne L, Beck JT, Glaspy JA, Peguero JA, Pluard TJ, Dhillon N, Hwang LC, Nangia C, Mayer IA, Meiller TF, Chambers MS, Sweetman RW, Sabo JR, Litton JK (2017) Prevention of everolimus-related stomatitis in women with hormone receptor-positive, HER2-negative metastatic breast cancer using dexamethasone mouthwash (SWISH): a single-arm, phase 2 trial. Lancet Oncol 18(5):654-662. https://doi.org/10.1016/S1470 -2045(17)30109-2

29. Assi H, Abdel-Samad N (2014) Severe gastrointestinal hemorrhage during targeted therapy for advanced breast carcinoma. Curr Oncol 21(5):e732-735. https://doi.org/10.3747/co.21.2038

30. Fujihara S, Mori H, Kobara H, Suenaga T, Hayashida Y, Sugimoto M, Kakehi Y, Masaki T (2013) Life-threatening gastrointestinal bleeding during targeted therapy for advanced renal cell carcinoma: a case report. BMC Nephrol 14:141. https://doi. org/10.1186/1471-2369-14-141

31. Gonzales P, Klusewitz S, Marowske J, Gancayco J, Osswald MB, Setlik R (2017) Everolimus implicated in case of severe gastrointestinal hemorrhage. Case Rep Oncol Med 2017:3657812. https ://doi.org/10.1155/2017/3657812

32. Shohdy KS, Lasheen S, Kassem L, Abdel-Rahman O (2017) Gastrointestinal adverse effects of cyclin-dependent kinase 4 and 6 inhibitors in breast cancer patients: a systematic review and meta-analysis. Ther Adv Drug Saf 8(11):337-347. https://doi. org/10.1177/2042098617722516

33. Hortobagyi GN, Stemmer SM, Burris HA, Yap YS, Sonke GS, Paluch-Shimon S, Campone M, Blackwell KL, Andre F, Winer EP, Janni W, Verma S, Conte P, Arteaga CL, Cameron DA, Petrakova K, Hart LL, Villanueva C, Chan A, Jakobsen E, Nusch A, Burdaeva O, Grischke EM, Alba E, Wist E, Marschner N, Favret AM, Yardley D, Bachelot T, Tseng LM, Blau S, Xuan F, Souami F, Miller M, Germa C, Hirawat S, O'Shaughnessy J (2016) Ribociclib as first-line therapy for hr-positive, advanced breast cancer. N Engl J Med 375(18):1738-1748. https://doi.org/10.1056/ NEJMoa1609709

34. Janni W, Alba E, Bachelot T, Diab S, Gil-Gil M, Beck TJ, Ryvo L, Lopez R, Tsai M, Esteva FJ, Aunon PZ, Kral Z, Ward P, Richards P, Pluard TJ, Sutradhar S, Miller M, Campone M (2018) First-line ribociclib plus letrozole in postmenopausal women with $\mathrm{HR}+$, HER2- advanced breast cancer: tumor response and pain reduction in the phase 3 MONALEESA-2 trial. Breast Cancer Res Treat 169(3):469-479. https://doi.org/10.1007/s10549-017-4658-x
35. O'Shaughnessy J, Petrakova K, Sonke GS, Conte P, Arteaga CL, Cameron DA, Hart LL, Villanueva C, Jakobsen E, Beck JT, Lindquist D, Souami F, Mondal S, Germa C, Hortobagyi GN (2018) Ribociclib plus letrozole versus letrozole alone in patients with de novo HR +, HER2- advanced breast cancer in the randomized MONALEESA-2 trial. Breast Cancer Res Treat 168(1):127-134. https://doi.org/10.1007/s10549-017-4518-8

36. Hwang JP, Somerfield MR, Alston-Johnson DE, Cryer DR, Feld JJ, Kramer BS, Sabichi AL, Wong SL, Artz AS (2015) Hepatitis $B$ virus screening for patients with cancer before therapy: American Society of Clinical Oncology provisional clinical opinion update. J Clin Oncol 33(19):2212-2220. https://doi.org/10.1200/ JCO.2015.61.3745

37. AISF Gestione clinica della Epatite B negli immunocompromessi 2017. http://www.webaisf.org/media/37755/gestione_clinica_ della_epatite_b_negli_immunocompromessi.2017.pdf. Accessed 31 Aug 2018

38. Barni S, Petrelli F, Cabiddu M (2016) Cardiotoxicity of antiemetic drugs in oncology: an overview of the current state of the art. Crit Rev Oncol Hematol 102:125-134. https://doi.org/10.1016/j.critr evonc.2016.04.012

39. Olasinska-Wisniewska A, Olasinski J, Grajek S (2014) Cardiovascular safety of antihistamines. Postepy Dermatol Alergol 31(3):182-186. https://doi.org/10.5114/pdia.2014.43191

40. Porta-Sanchez A, Gilbert C, Spears D, Amir E, Chan J, Nanthakumar K, Thavendiranathan P (2017) Incidence, diagnosis, and management of QT prolongation induced by cancer therapies: a systematic review. J Am Heart Assoc. https://doi.org/10.1161/ jaha.117.007724

41. Freedman RA, Tolaney SM (2018) Efficacy and safety in older patient subsets in studies of endocrine monotherapy versus combination therapy in patients with HR +/HER2- advanced breast cancer: a review. Breast Cancer Res Treat 167(3):607-614. https ://doi.org/10.1007/s10549-017-4560-6

42. Kovarik JM, Beyer D, Bizot MN, Jiang Q, Shenouda M, Schmouder RL (2005) Blood concentrations of everolimus are markedly increased by ketoconazole. J Clin Pharmacol 45(5):514518. https://doi.org/10.1177/0091270005275368

43. Samant TS, Dhuria S, Lu Y, Laisney M, Yang S, Grandeury A, Mueller-Zsigmondy M, Umehara K, Huth F, Miller M, Germa C, Elmeliegy M (2018) Ribociclib bioavailability is not affected by gastric $\mathrm{pH}$ changes or food intake: in silico and clinical evaluations. Clin Pharmacol Ther 104(2):374-383. https://doi. org/10.1002/cpt.940

Publisher's Note Springer Nature remains neutral with regard to jurisdictional claims in published maps and institutional affiliations. 